\title{
On the Brink of Shifting Paradigms, Molecular Systems Engineering Ethics Needs to Take a Proactive Approach
}

\author{
Raheleh Heidaria ${ }^{a}$ Bernice S. Elger ${ }^{a}$, and Ralf Stutzki ${ }^{\star b}$
}

\begin{abstract}
Molecular Systems Engineering (MSE) is a paradigm shift in both engineering and life sciences. While the field is still in its infancy the perspectives of MSE in revolutionising technology is promising. MSE will offer a wide range of applications in clinical, biotechnological and engineering fields while simultaneously posing serious questions on the ethical and societal aspects of such technology. The moral and societal aspects of MSE need systematic investigation from scientific and social perspectives. In a democratic setting, the societal outcomes of MSE's cutting-edge technology need to be consulted and influenced by society itself. For this purpose MSE needs inclusive public engagement strategies that bring together the public, ethicists, scientists and policy makers for optimum flow of information that maximizes the impact of public engagement. In this report we present an MSE consortium and its ethics framework for establishing a proactive approach in the study of the ethics of MSE technology.
\end{abstract}

Keywords: Art of molecule - Ethics - Molecular systems engineering - Proactive ethics - Public engagement . Society

\section{Molecular Systems Engineering}

The origin of life, i.e. how early life form(s) appeared on earth, is still a mystery not only for science but also for philosophy. In 2014, scientists simulated an extra-terrestrial high-power strike that triggered the synthesis of nucleobases - the ingredients of life - from formamide. ${ }^{[1]}$ Formation of nucleobases from formamide about 4 billion years ago is one of the possible scenarios that could explain the sudden emergence of RNA (ribonucleic acid) as the earliest precursor of life. Deciphering the processes of abiogenesis potentially enables scientists to write their own recipe of genetic code and re-create exotic life forms as not seen before. The emergence of interdisciplinary fields such as synthetic biology, together with systems approaches in discovery science (e.g. systems chemistry and systems biology) are revolutionizing our traditional attitude in the life sciences and open, as yet unprecedented, possibilities to develop novel applications in medical science, pharmaceutical and agricultural industries.

${ }^{\star}$ Correspondence: Dr. R. Stutzki E-mail: ralf.stutzki@unibas.ch alnstitute for Biomedical Ethics University of Basel

${ }^{b}$ NCCR Molecular Systems Engineering

University of Basel

Klingelbergstrasse 80, CH-4056 Basel
Synthetic biology takes advantage of multiple problem-solving strategies: it permits us to determine a minimal gene setting that constitutes a living cellular form (top-down approach), and to construct synthetic biological systems, protocells and living cells (bottom-up approach) with the ultimate goal: to redesign life. ${ }^{[2]}$ Systems biology integrates this information into computational models and mathematical formulae that can describe the structure of the system. ${ }^{[3]}$ The bottom-up approach of synthetic biology in the construction of living cells/organ-

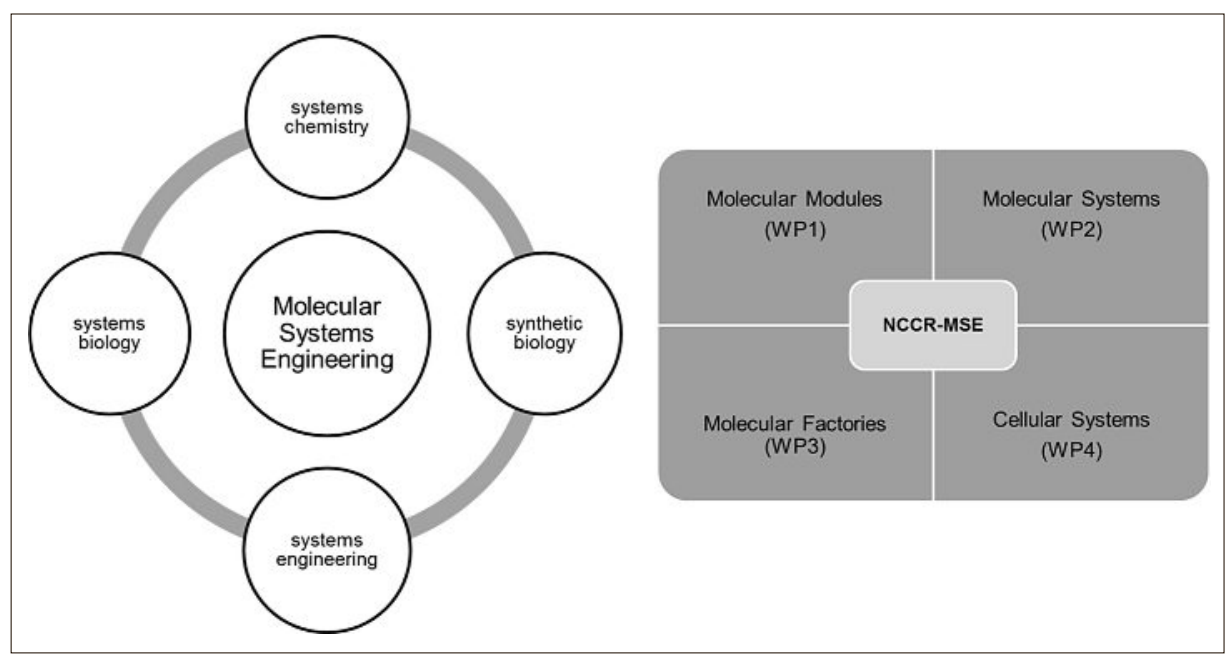

Fig. 1. a) MSE as an interdisciplinary subject: Molecular Systems Engineering is built upon several interrelated disciplines. It applies engineering principles to redesign molecular modules that can be integrated into more complex systems and exploit their functionality for the desired purposes. b) Structure of the NCCR-MSE work-packages: the four work-packages break down the MSE mission into concrete deliverables across 26 individual projects. 
Molecular systems engineering (MSE) applies engineering principles towards design and development of novel molecular modules with programmable structure and predictive behaviour. These functional molecular modules can be integrated into complex systems such as cells to exploit the system for purposes not necessarily seen in nature. MSE is a cutting-edge concept inspired by nature that holds high hopes for addressing major challenges in the fields of biomedical science, drug discovery, energy, environment and engineering.

Molecular systems engineering is a paradigm shift in the design of natural or synthetic systems. The ability to program and adjust molecular modules opens up enormous opportunities to develop new drugs, diagnostic tools, fuels, plants and other organisms. For instance, one major challenge in drug discovery is to enhance the efficacy of drug delivery to the target tissue in order to limit its unwanted side effects in non-target tissue; scientists have recently been able to engineer a cellular Trojan horse by optimizing polymer nanoreactors that release the desired material within the limits of a target cell, upon demand. ${ }^{[5]}$ Such cellular nanoreactors can be inserted into a cancerous or infected cell to dictate its programmable death and hence prevent further metastasis of cancer or spread of the infection. Other scientists have developed implantable capsules for type 1 diabetes that contain two different molecular modules, a high-precision $\mathrm{pH}$ sensor that measures the blood $\mathrm{pH}$ and when it detects the pathologic $\mathrm{pH}$ specific to diabetes (diabetic ketoacidosis $\mathrm{pH}<$ 7.1 ), an activating signal will be relayed to the second module containing genetically-engineered cells that release insulin only in response to the signal received from the first module. This molecular module is a closed-loop prosthetic network which is controlled by $\mathrm{pH}$ level; hence physiological $\mathrm{pH}$ switches off insulin secretion. ${ }^{6]}$ There are endless possibilities for multidisciplinary inventions using molecular modules; as the field is evolving rapidly we are stepping into an as yet unknown realm of technology, where a changing landscape poses big questions on the societal dimensions of such inventions.

In this report we review major societal and ethical challenges of MSE and we discuss how a proactive approach towards cutting-edge technology contributes to the minimization of risks while facilitating the necessary balancing of risks and benefits in a socially accepted way. For a concrete discussion we present a large-scale MSE consortium framed as the Swiss National Centre of Competence in ResearchMolecular Systems Engineering (NCCRMSE) to develop our practical model of social science and ethics committees in such interdisciplinary multi-stakeholder partnerships.

\section{NCCR: A Model of a Molecular Systems Engineering Consortium}

Worldwide, there have been several consortia for developing synthetic biology, however molecular systems engineering adds an extra dimension to synthetic biology by engineering molecules as intelligent modules that are capable of integrating into complex cellular systems. NCCRMSE, which started operating in 2014 is an interdisciplinary research project in Switzerland funded by the Swiss National Science Foundation. The project directed by the University of Basel and ETH Zurich currently brings together more than 100 researchers from 9 institutes to realize the ambitious goal of molecular systems engineering. The consortium is comprised of, at present, 26 individual projects embedded within 4 work packages (WPs) (Fig. 1b). WP1 takes the first step to develop efficient building blocks upon which molecular modules are founded. To assemble molecular modules into cellular systems they must be structurally adaptable to the cell's media, therefore this work package investigates novel molecular transporters, artificial enzymes, cellular matrices and all the machinery required for integration of a molecular module. The dynamic of such modules and how they will be transported to their destination is the focus of WP2, which aims at assembling and characterizing molecular systems from molecular modules. Next, WP3 is set out to assemble molecular modules and molecular systems to create multifunctional systems resembling molecular factories. For this purpose, it optimizes the architecture, topology and evolvability of the modules within a more complex system. Finally, the nine project groups in WP4 take up the challenge to incorporate these intelligent molecular modules into cellular systems either at cell, tissue or organ levels. ${ }^{[7]}$

\section{Ethical Dimensions of MSE}

Molecular systems engineering intends to offer solutions to fundamental problems by integrating different perspectives. For instance, by implementing mathematics, physics and chemistry approaches in biology, MSE can resolve some of the basic challenges of drug discovery. However, MSE will not restrict its application to biomedical science. It will also open new windows to the environmental sciences and introduce a new era of synthetic biology and synthetic chemistry. While the novelty of this interdisciplinary field keeps fascinating scientists, a more hidden aspect of such an enterprise may end up causing unanticipated social bewilderment. We can already observe the somewhat disconcerted reactions to a new emerging dimension of MSE that concerns the application of designer molecular modules in medicine, pharmacological products, agriculture, environment, fuel, energy resources and even in the form of new living organisms. Inventions that involve the reprogramming of cells and organisms or artificial design of biosynthetic modules or, even more adventurous, when different synthetic modules are assembled to form an intelligent multi-compartment system, these new entities and means to modify nature have been subject to ethical considerations that span a wide variety of concerns. However, these are only the tip of the iceberg and many consequences remain unknown for now. From a consequentialist point of view, the ethics of MSE highly depends on a favourable ratio between the risks and the benefits, i.e. potential outcomes such as new diagnostic tools, therapeutics, technological devices, bacterial forms and plants are crucial. To further appropriate a rational evaluation of these new technologies and to avoid barriers due to uninformed fears and overreactions, a proactive approach in timely recognition of ethical challenges will accelerate the transition of such cutting-edge technologies into relevant sectors that benefit society.

By implementing a proactive approach towards interdisciplinary fields, 'Molecular Systems Engineering Ethics' (MSEE) creates a framework that is helping to identify problems on the rise and collect information for a comprehensive assessment of the risks and benefits to develop the best course of action. One advantage of a proactive approach is that MSEE does not need to wait for negative consequences to emerge; on the contrary, it is able to point towards and to avoid such problems by developing preventative strategies. Fig. 2 summarizes the benefits of a proactive approach in research ethics. Proactive ethical reflections related to MSE need to deal with two major types of problems: first, concrete problems that are customary in science fields and are usually addressed by regulatory frameworks and code of conducts. These include issues of intellectual property (IP), rights of exploitation of the results for different partners (e.g. patent issues); equal opportunity issues in recruitment of scientists, justice in distribution of research outcomes and analysis of population groups who benefit from the inventions, bioethics concerns such as use of stem cells, human embryo, use of animals and so on. Several of the concrete problems have been identified in other areas of science and are, in part, already well-studied. By contrast, the 
second type of problem remains abstract: a proactive ethics approach needs to explore the nature of inventions, products and technologies made or expected to be made by MSE. It is an attempt to decipher multiple dimensions of the inventions to identify the relationship between the research outcomes and their societal impacts. By abstract problems, we refer to moral and ethical aspects of MSE's capacity and its potential future outcomes. For instance; one promise of MSE is to invent next-generation diagnostic self-testing tools for home or smartphone devices. The potential of such devices is nearly unlimited; it can be a self-testing kit for cancer diagnosis, HIV infection or any other life-threatening situation. Such inventions revive ethical concerns at multiple layers. MSE could design smart microbial modules that consume pollution and keep the environment fresh while at the same time they may pose biosafety threats to the ecosystem and human life. It is important to note that the proactive approach of MSEE is not restricted to foreseeing negative consequences of new inventions; perhaps a more important aspect of MSEE is to identify and make palpable the positive outcome of MSE, meaning that MSEE could actively bridge the cutting-edge technology of molecular systems engineering to social structures such as health care systems and education where they can be best implemented to benefit society. MSEE's proactive strategy could thus be the initial step towards introducing the capacity and opportunities of new age technology to society and offer the opportunity of direct interaction between scientists, policy makers, ethicists and the public to discuss the potential outcomes of MSE and to evaluate their impact from diverse perspectives.

Another advantage of such a proactive ethical approach is that the research consortium has the opportunity to apply ethical principles at all stages of the program; from planning and conducting, to the dissemination level. To establish a constructive partnership in the context of interdisciplinary projects, MSEE and scientists need to inaugurate a strong communication medium where the primary necessity is to develop a shared language of which both parties have full comprehension. While research ethics committees that evaluate human subject research once the technology is first tested in humans are important, there is also a need for other types of ethics forums and committees. MSEE scientists involved in the composition of large-scale research projects such as NCCR-MSE have the responsibility to not only introduce the principles of research ethics to their partner stakeholders, but also to ensure that scientists, managerial teams and ethicists implement adequate and precise

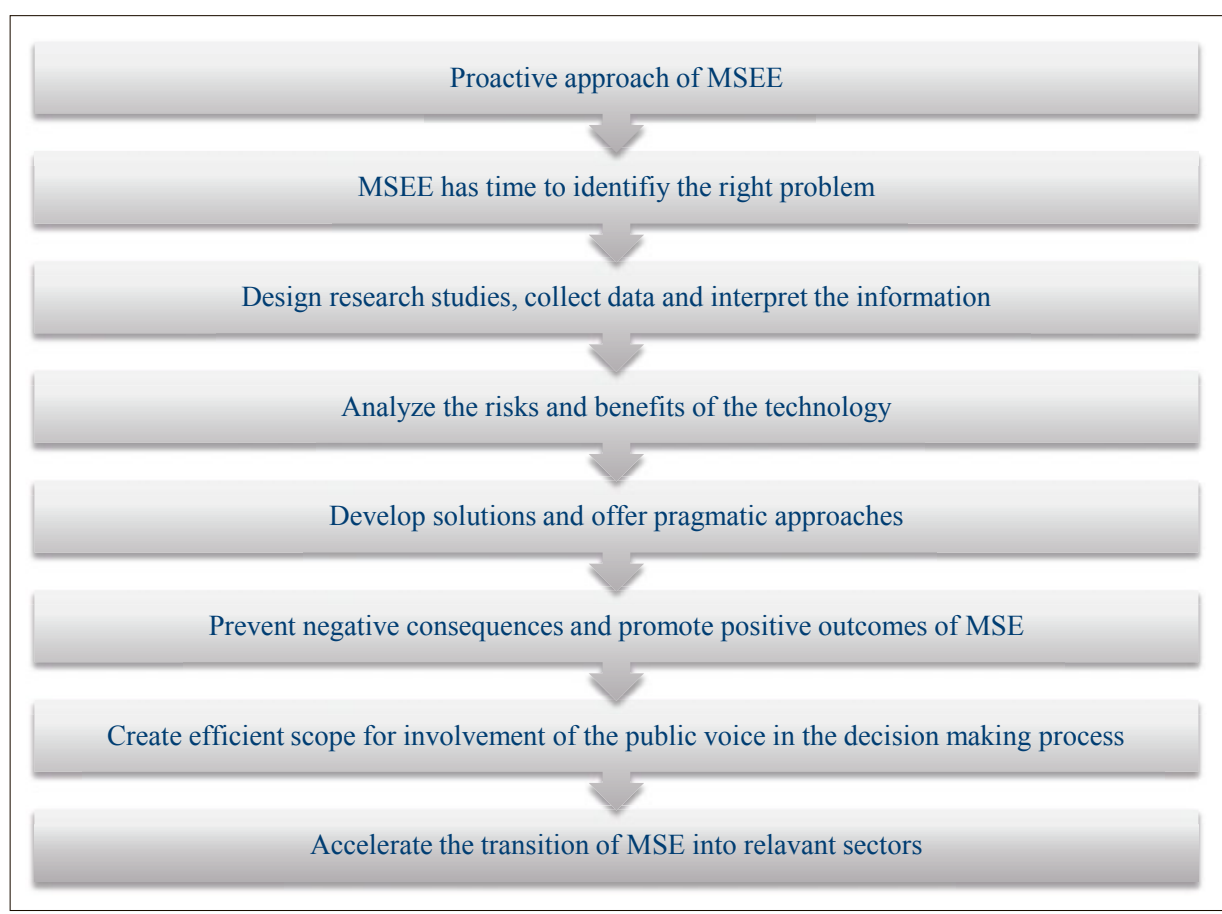

Fig. 2. An overview of a proactive approach in the ethics of MSE and its impact on the transition of MSE into relevant sectors.

terminology when disseminating their results in a social context. This initial harmonization will help reduce tensions and misunderstanding at the translational level. Similarly, such new types of ethics forums or committees that are involved in buffering social consequences of cutting-edge technology should develop a consistent connection with scientists in order to sustain a realistic measurement of risks and benefits that does not discourage scientific advancement, while holding strong to a commitment to ethical principles of scientific research.

\section{Research Integrity and Public Engagement}

We are living in the age of technology, the accelerating pace of research and development has opened up new horizons of scientific capabilities. Now that information technology has facilitated access to science resources, a higher level of public engagement in scientific programs is anticipated. Public engagement is a broad concept that entails public involvement at different levels. One popular interpretation of public engagement is based on the 'flow of information' model, in which three main activities constitute the public engagement including public communication, public consultation and public participation. The flow of information model refers to " $\max$ imizing the relevant information (knowledge and/or opinions) from the maximum number of relevant sources and transferring this efficiently to the appropriate receivers". ${ }^{[8]}$ As implied by the definition, 'information' is the essential component that is in constant exchange between sponsors of an initiative and the public. Therefore, a primary step is to enhance the transfer of information to the public, the integrity and transparency of this information are crucial as public perception will be a reflection of this exchanged information that ultimately constitute the public participation. Table 1 presents a short description of the three activities and relevant examples in a typology context of public engagement. Perhaps public engagement in different socio-cultural backgrounds requires tailored communication, consultation and participation strategies that promote highest effectiveness? Also, each of these methods need to be adjusted to the context of the research program; for instance in case of MSE, ethics committees together with scientists need to design the engagement mechanisms that best match the evolving concept of molecular systems engineering. This includes outreach activities to convey the information about MSE, its implications, risks and benefits and consequently investigate the public reflection on this matter, solicit their opinion and put them in relevant practice. Although a unique formulation for public engagement in different research fields may appear unrealistic, what remains consistent is the importance of societal engagement in policy-making processes. Practically, societal engagements may raise conceptual impacts by influencing people's knowledge, understanding and attitudes towards an issue or may result in instrumental impacts by influencing policy, regulations or behaviour of the society towards certain 
issues. ${ }^{[9]}$ Even if effective engagement mechanisms are incorporated in a research program, still further investigations are required to measure the policy impact of public engagement. Maximizing the policy impact of society's reflection on sensitive subjects such as science and technology not only serves the democratic constitution of the society, where tax payers are actively involved in decision making, but also prepares a socio-cultural context for translation of cutting-edge technologies.

One risk concerning public engagement is misrepresentation of science that could occur at any level of communication from institution to the news chain; one comprehensible example is science hyping that overinflates the public expectation of science by misinterpreting a scientific discovery or exaggerating potentials of current technology. Failure of science with this regard not only diminishes public trust but also desensitizes the audience to real scientific breakthroughs. ${ }^{[10]}$ Conceivably, introducing a timely standard of conduct for multi-partnership research projects such as the NCCR-MSE will consolidate the research integrity amongst scientists and other stakeholders while remaining faithful to public trust. When talking about ethical considerations of interdisciplinary endeavours we need to keep in mind that defining ethical norms in a scientific context vastly depends on the perspective and professional traditions of each discipline (synthetic biology, chemistry, engineering, etc.), therefore a harmonization of these norms promote research integrity while preventing future conflicts. Main conceptual inputs in defining research ethics norms could be provided for example by

ethicists, scientists and the media. But it must be kept in mind here that these three groups, while grounded in society, do not represent society with its values and norms at large. This again rephrases the importance of direct engagement of the public in redefining research norms that eventually affect the policy-making process (Fig. 3).

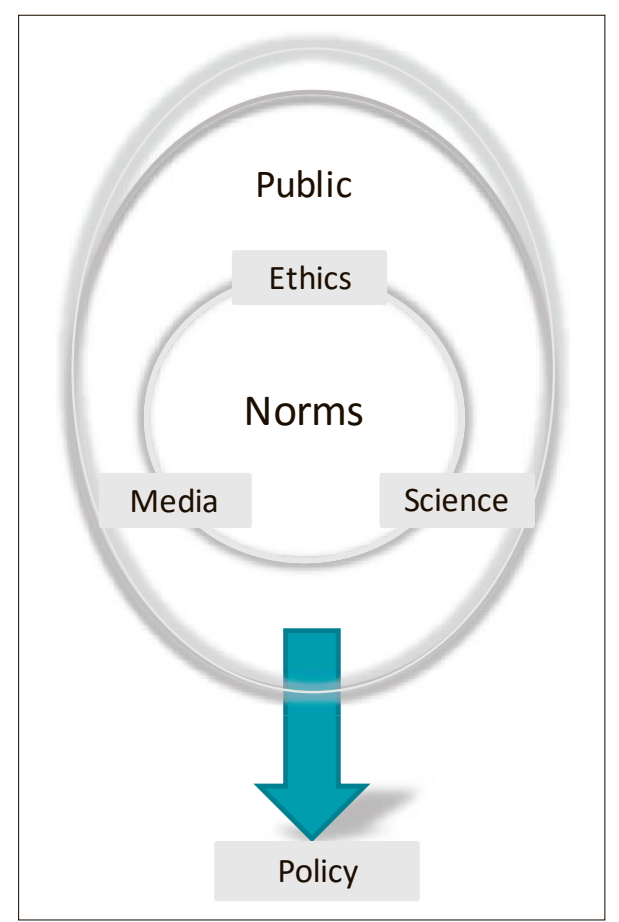

Fig. 3. The public exerts its influence on the policy making process through its impact on media, science and ethics in defining ethical norms; however, a more direct impact of the public's influence in defining norms is lacking. Existing policies also mutually influence public perception of technology and shapes the social recognition of ethical norms.

Table 1. The three main mechanisms of public engagement strategies. The flow of information model implements communication, consultation and participation methods for defining the concept of public engagement. Source: reproduced with permission from the authors. [8]

\section{Type of public engagement mechanism}

Public Communication

Public Consultation

\section{Description}

The flow of information is from the sponsors of the initiative to the public representatives

In a process initiated by the sponsors, public representatives convey the information to the sponsors

\section{Public Participation}

An exchange of information between representatives of the public and the sponsors. There is a dialogue and exchange of raw opinion between the two groups

\section{Examples}

Information broadcasts, public meetings, interactive internet information, hotline and...

Opinion poll, referendum, survey, voting, consultation document, interactive website, focus group, study circle, open space, citizen panel group based and...

Action planning workshop, citizen's jury, consensus conference, negotiated rule making, task force, deliberative opinion poll, planning cell, town meeting and..
In the next section, we introduce the structure of the NCCR-MSE ethics framework to discuss the proactive strategy in ethical assessment of MSE, show the public engagement mechanisms that are implemented therein to elicit public interest and give an overview of its role in opening up a dialogue between scientists and the public.

\section{Ethics of NCCR-Molecular Systems Engineering}

The NCCR Molecular Systems Engineering Ethics (NCCR-MSEE[11]) is a two branch endeavour: at the beginning of this research project the position of Head of Ethics was installed in Basel while simultaneously, integrated within WP4, an ethics team based at the University of Zurich started to investigate the societal aspects of this NCCR, along with its ethical hurdles and their long-term impact on policy and the economy. At this, still very early phase of the research project, we will focus here on the overall ethics strategy developed by the Head of Ethics (HE) in close cooperation with the NCCR directory board. ${ }^{[12]}$

In its first 15 months, the focus has been placed on building an ethics matrix that shall serve the NCCR-MSE as a reliable tool of ethical and moral-philosophical orientation in the years to come. Obviously, building such a matrix is an ongoing process; hence, the first results presented here can only be looked on as a work in process.

A shift in the engineering paradigm which is expected to be a central outcome of this research project automatically leads to significant ethical challenges which, if possible, ought to be filtered out and addressed in due time. But this is not a task to be completed solely by the ethicists involved in the NCCR-MSE nor, for that matter, by the scientists working there; rather and as shall be discussed, ethical analysis and decision-making is a process which calls for the involvement of society at large and it is here where ethics must pave the way and search for the appropriate channels and tools. By focussing on the development of communication platforms for researchers involved in this NCCR and for the public alike, the NCCR-MSEE is novel and distinct from traditional ethical analysis. NCCR-MSEE takes an integrative, proactive approach by

1) Supporting each individual project researcher whenever and wherever necessary;

2) Developing educational vessels like seminars for PhDs, Postdocs and project leaders;

3) Promoting interdisciplinarity within the scientific moral discourse and

4) Encouraging the non-scientific com- 
munity, in which this research is grounded to speak up and engage actively in all project-driven, (not only) controversial issues of moral relevance.

Bridging the ever-increasing gap not only between different fields of science but also between the scientific community and the public at large is the pivotal challenge of this research's ethics. In order to address this tremendous task in a systematic way we are currently developing a matrix which aims to foresee and guide through ethical crossroads that will appear along the road of this NCCR's research. We call this matrix 'Art of Molecule - die Art des Moleküls' (AoM), a headline in two languages - English and German. While this title may suggest the poor translation talent of its author, this irritation is calculated: the English branch of this brand invites the arts on a large scale to interpret, comment on and challenge molecular systems engineering whereas the German branch ('Art') calls for a philosophical reflection of molecular being ('Molekulares Sein') and as such, of life and being in itself. As already mentioned, these fundamental issues of concern belong to the societal, public field at large where within an open, yet guided, framework all parties at stake may enter into the discourse on par.

It is still too early to present a reflective analysis of the AoM matrix as this concept is still in its inception. Nevertheless, a able to develop and install so far are promising (Fig. 4):

\section{On-site visits of the NCCR-MSE work package leaders}

We have started to arrange on-site visits with the NCCR-MSE WP leaders to get an overview of the various and interdisciplinumber of endeavours that we have been

nary research projects and to connect on a personal level with them and their scientific teams. Such meetings are valuable for all parties involved since they not only provide insights into each other's work but also serve as a source of inspiration for the tasks which lie ahead.

\section{Ethics on Site (EoS)}

PhDs and Postdocs working in this NCCR have been invited to enrol and participate in this accredited seminar. In this course, NCCR's own as well as external ethics experts are invited to lecture and to encourage the participants to engage actively in the process of building an NCCR-MSE ethics framework. Ideas and input of the participants have already been numerous and are now gradually being introduced as part of the refinement of the AoM matrix.

\section{Curriculum}

An outline of an ethics curriculum - as part of the NCCR-MSE Master of Arts and $\mathrm{PhD}$ program that will be established in the near future - has been developed. It includes lectures and courses on applied ethics, research ethics as well as introductory seminars focusing on moral philosophical challenges of molecular systems engineering in general. Furthermore, a special focus has been laid on the development of soft skills seminars such as 'rhetoric and public speech', the mastery of which undisputedly is indispensable in any process requiring ethical discourse.

\section{COMSEE}

The NCCR-MSE currently embraces more than 100 scientists representing various academic disciplines, different cultures and diverse research institutions

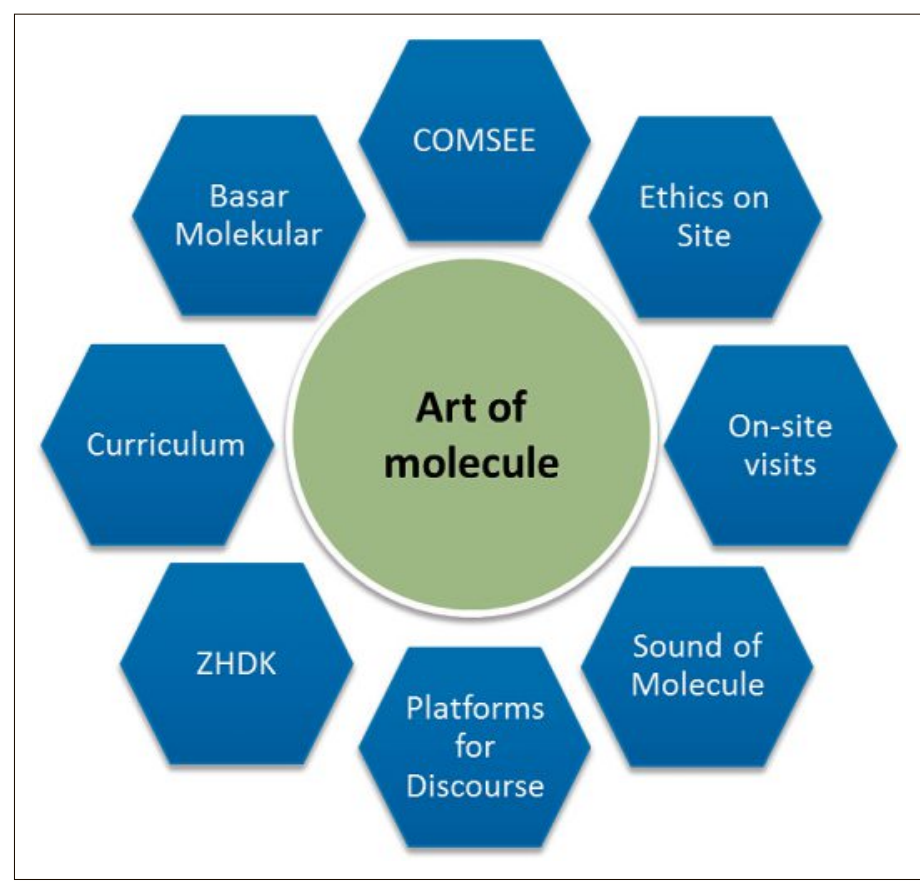

spread all over the country. Undoubtedly, such variety is of great value for any scientific endeavour. But it also calls for a discussion about the content of value systems and guiding tools each and every member of the research team applies amidst ethical conflict. Given the diversity of research disciplines and researchers involved in this scientific project it is more than likely that the existing catalogue of moral and philosophical tools must be harmonized in order to function properly. The NCCR-MSEE along with the directory board recognizes the need to develop a 'Code of Molecular Systems Engineering Ethics' (COMSEE) in the future, which will be of binding character to everyone involved.

\section{Platforms for Discourse}

Ethical aspects of molecular systems engineering must be openly elaborated internally and externally. This requires all agents' ability and will as well as the indispensable conditions for communication on par, particularly between the sciences and the non-scientific interested public. While an interdisciplinary exchange is a most valuable and even essential contribution to the evolving field of molecular systems engineering, it must be acknowledged by the scientists involved that this research is grounded and embedded within society which - as is the case with NCCRMSE - also finances this project. Science must inform its community about research goals, work progress as well as unexpected difficulties which may arise in the course of any research. Accordingly, appropriate platforms for discourse must be developed.

\section{Basar Molekular}

A first step towards creating the conditions for a discourse on par has been the installation of the radio broadcast 'Basar Molekular'. This public, live event takes place twice a year and is being broadcast - in German - by three non-commercial radio stations in Switzerland. We invite scientists involved in the NCCR-MSE as well as public figures and opinion leaders from non-scientific areas of society to talk with each other amidst a live audience, which is also encouraged to participate in the discussion. Inspired by the 'Vienna School' at the beginning of the $20^{\text {th }}$ century where scientists, artists, philosophers, etc. inspired each other's work by simply entering into a dialogue and exchanging thoughts and ideas, the members of this NCCR also enter into a constructive, critical dialogue with society in general and its representatives. Basar Molekular can also be listened to via live stream on the Internet. 


\section{Sound of Molecule}

Representing one of the pillars of the AoM matrix 'Sound of Molecule' invites - amongst other musicians - the renowned Swiss classical orchestra 'Argovia Philharmonic' to interpret artistically the concepts of molecular systems engineering. This challenging cooperation is not set out to mount musical interpretations and/or compositions of the NCCR-MSE research endeavour - even though this may be one outcome; rather, the parallelism of both scientific and artistic creative power - including the variety of their inherent difficulties - will be in the focus of interest here.

\section{ZHDK}

Recently, a cooperation between the NCCR-MSEE and a branch of the Zurich University of Arts (ZHDK) has been established. A group of ZHDK Master students along with their teachers from diverse artistic departments will be invited to connect with this NCCR's work packages and to enter into an open dialogue with the stakeholders. Learning processes and an exchange of ideas - on both sides - shall foster a unique and fruitful interdisciplinarity between the arts and the sciences in the coming years. It is hoped that innovative perspectives, new interpretations, inspiration, answers and solutions will strengthen the ethical discourse of the NCCR-MSE.

\section{Conclusions}

Molecular systems engineering is catalysing a paradigm shift in both engineering and life sciences. This endeavour undoubtedly poses significant ethical and societal challenges that need to be studied systematically. In this report we have proposed that ethical and societal questions in respect to the consequences of MSE are a social matter that requires a new proactive approach that goes beyond traditional ethical analysis. It requires active involvement of not only scientists, ethicists and MSE stakeholders but also of the public, as the aim of this process is to favour ground-breaking policy making decisions that potentially affect major social realities. We have presented the integrative approach of MSEE in providing education, research ethics and individual support as well as pragmatic strategies to promote public engagement in project-driven controversial issues. NCCR-MSEE has created an innovative matrix of an ethical 'Art of Molecule' that implements multiple educational, social and entertaining activities to raise the public interest in the emerging field of molecular systems engineering. Although the NCCR-MSE is a relatively new consortium, the proactive approach of MSEE provides a solid background for pinpointing the positive outcomes while incorporating preventive solutions to both foresee and thus be enabled to avoid negative consequences. While MSE holds high hopes for new-age technology, the ethical and societal aspects of it also need to be meticulously considered to fulfil the promise of science.

\section{Acknowledgments}

This publication is supported by the Swiss National Science Foundation as part of the NCCR Molecular Systems Engineering.

Received: March 11, 2016

[1] M. Ferus, D. Nesvorny, J. Sponer, P. Kubelik, R. Michalcikova, V. Shestivska, J. E. Sponer, S. Civis, Proc. Natl. Acad. Sci. USA 2015, 112, 657.

[2] S. A. Benner, A. M. Sismour, Nature Rev. Genetics 2005, 6, 533.

[3] T. G. Trey Ideker, Leroy Hood, Ann. Rev. Genomics and Human Genetics 2001, 2, 343.

[4] G. Von Kiedrowski, S. Otto, P. Herdewijn, $J$. Systems Chem. 2010, 1,1

[5] P. Baumann, M. Spulber, I. A. Dinu, C. G. Palivan, J. Phys. Chem. B 2014, 118, 9361.

[6] D. Auslander, S. Auslander, G. Charpin-El Hamri, F. Sedlmayer, M. Muller, O. Frey, A. Hierlemann, J. Stelling, M. Fussenegger, Molecular Cell 2014, 55, 397.

[7] I. E. Holm, A. K. Alstrup, Y. Luo, J. Pathol. 2015, 238, 267.

[8] G. Rowe, L. Frewer, Sci., Technol. Human Values 2005, 30, 251.

[9] J. Phillipson, P. Lowe, A. Proctor, E. Ruto, $J$. Environ. Manag. 2012, 95, 56.

[10] A. Rinaldi, EMBO Rep. 2012, 13, 303.

[11] MSE refers to molecular systems engineering and MSEE refers to molecular systems engineering ethics.

[12] 'MSE Ethics' and 'NCCR-MSEE' in this paper thus refer exclusively to the work of the Head of Ethics (HE). 